\title{
Evaluation and predictive factors analyses for patient-self-reported depression, anxiety and quality of life in patients with metastatic renal cell carcinoma underwent interferon- $\alpha$ treatment: a prospective cohort study
}

\author{
Yanjie Wang, Bingbing Song, Yuling Zhang, Haibo Li \\ Department of Urinary Surgery, Harbin Medical University Cancer Hospital, Harbin 150000, China \\ Contributions: (I) Conception and design: H Li; (II) Administrative support: None; (III) Provision of study materials or patients: Y Wang; \\ (IV) Collection and assembly of data: B Song; (V) Data analysis and interpretation: Y Zhang; (VI) Manuscript writing: All authors; (VII) Final \\ approval of manuscript: All authors. \\ Correspondence to: Haibo Li. Department of Urinary Surgery, Harbin Medical University Cancer Hospital, 150 Haping Road, Harbin 150000, China. \\ E-mail: lihaibo16@126.com.
}

Background: This study aimed to investigate the patient-self-reported depression, anxiety and quality of life (QoL) as well as their predictive factors in patients with metastatic renal cell carcinoma (mRC) underwent interferon-alpha (IFN- $\alpha)$ treatment.

Methods: A total of 127 patients with mRCC underwent IFN- $\alpha$ treatment were consecutively enrolled in this prospective cohort study. Anxiety, depression and QoL were assessed at week 0 (W0), W4, W8 and W12 by Hospital Anxiety and Depression Scale (HADS) and European Organization for Research and Treatment of Cancer Quality of Life Questionnaire (EORTC QLQ-C30 Scale), respectively.

Results: The percentages of anxiety and depression both increased at W12 compared with W0. The EORTC QLQ-C30 Scale-Global health status score, EORTC QLQ-C30 Scale-Function and EORTC QLQ-C30 Scale-Symptoms scores did not deteriorate at W4, W8 or W12 compared with W0. Multivariate logistic regression analyses revealed that older age and elevated level of highest education independently predict reduced risk of HADS-Anxiety score elevation at W12, while hypertension was an independent predictive factor for increased risk. Older age also independently associated with less risk of elevated HADSDepression score. Smoke, hypertension, diabetes and higher ECOG performance were independent predicting factors for escalated risk of EORTC QLQ-C30 Scale-Global health status score elevation at W12, and higher ECOG performance was an independent predicting factor for increased risk of elevated EORTC QLQ-C30 Scale-Function score at W12.

Conclusions: In conclusion, anxiety and depression increased while QoL did not deteriorate in mRCC patients during IFN- $\alpha$ treatment, and age, highest education, hypertension, smoke, diabetes as well as higher ECOG performance status correlated with their aggravation.

Keywords: Metastatic renal cell carcinoma (mRCC); interferon-alpha; anxiety; depression; quality of life; predictive factor

Submitted Feb 26, 2018. Accepted for publication Jul 31, 2018.

doi: $10.21037 /$ tcr.2018.11.07

View this article at: http://dx.doi.org/10.21037/tcr.2018.11.07 


\section{Introduction}

Renal cell carcinoma (RCC), the ninth most frequent carcinoma in males all over the world according to the report of Global Cancer Statistics in 2012, presents with an escalating incidence in the past decade worldwide especially in more developed countries (1-3). In China, there were estimated 668,000 new RCC cases in 2015 consisting of 432,000 male patients and 236,000 female patients, and 234,000 RCC related deaths including 152,000 male cases as well as 82,000 female cases (4). Among all RCC cases, metastatic RCC (mRCC) accounts for approximately $30 \%$ of all RCC cases, for which the treatment goal is to relieve symptoms and prolong survival (5). Although the treatment options for $\mathrm{mRCC}$ has altered from comprehensive immunosuppressive therapy to targeted therapy and new immunosuppressive drugs, due to good treatment responses observed by clinical studies, interferon-alpha (IFN- $\alpha$ ) is still the most commonly applied drug in mRCC patients $(6,7)$.

However, the problems concerning psychiatric status and quality of life (QoL) in mRCC patients treated by IFN- $\alpha$ are drawing increasing attention among oncologists due to that a growing number of mood disorders and change of QoL have been reported $(8,9)$. Unstable psychiatric status in mRCC patients mainly refers to anxiety and depression, which could be evaluated by numerous scales such as Hospital Anxiety and Depression Scale (HADS), a selfassessed scale generally applicable in cancer patients $(10,11)$. As another crucial aspect, QoL is already extensively assessed in patients with other malignancies for the purpose of evaluating and improving symptoms, physical function and life satisfaction after various treatments including IFN- $\alpha$ therapy (12-14). Unfortunately, insufficient clue for reducing the risk of depression, anxiety and decrease of QoL in mRCC patient through identifying the predicting factors is accessible for clinicians until now.

Thus, the aim of our study was to investigate the patientself-reported depression, anxiety and QoL as well as their predictive factors in patients with mRCC underwent IFN- $\alpha$ treatment.

\section{Methods}

\section{Participants}

A total of 127 patients with mRCC underwent IFN- $\alpha$ treatment at the Department of Urinary Surgery in Harbin Medical University Cancer Hospital between Jan 2013 and Dec 2016 were consecutively enrolled in this prospective cohort study. Inclusion criteria were as follows: (I) diagnosed as mRCC according to clinical, imaging and pathological findings; (II) age above 18 years; (III) about to receive IFN- $\alpha$ treatment; (IV) life expectancy longer than 12 months; (V) able to be followed up regularly and complete the assessment questionnaires. Patients were excluded if they (I) had brain metastasis; (II) accompanied with other solid tumors or hematological malignance; (III) had uncontrolled hypertension, severe infection or primary organ failure; (IV) were pregnant or lactating, or planned for pregnancy.

\section{Ethics}

This study was approved by the Ethics Committee of Harbin Medical University Cancer Hospital and conducted according to the Helsinki Declaration. The number of the Ethic Approval was KY2012-12. All patients provided written informed consents.

\section{Baseline data collection}

After the enrollment, patients' baseline characteristics were collected, which included age, gender, highest education, body mass index (BMI), overweight, smoke, drink, hypertension, hyperlipidemia, diabetes, Eastern Cooperative Oncology Group (ECOG) performance, histology, number of metastatic sites, location of metastatic sites, tumor size, prior radiotherapy and prior chemotherapy.

\section{Treatment}

This prospective cohort study did not intervene treatments of patients. All patients underwent IFN- $\alpha$ treatment "on demand" according to disease conditions and patients willingness. The common treatment was as follows: $9 \mathrm{MU}$ IFN- $\alpha$ by subcutaneous injection 3 times/week for 12 weeks.

\section{Assessments of anxiety and depression}

The anxiety and depression of patients were assessed at week 0 (W0), W4, W8 and W12 by Hospital Anxiety and Depression Scale (HADS) anxiety (HADS-Anxiety) score and depression (HADS-Depression) score. Both HADSAnxiety and HADS-Depression consist of seven questions which were scored as $0-3$ points individually, resulting in $0-21$ points and being classified as: $0-7$, no anxiety/ depression; 8-10, light anxiety/depression; 11-14, moderate 


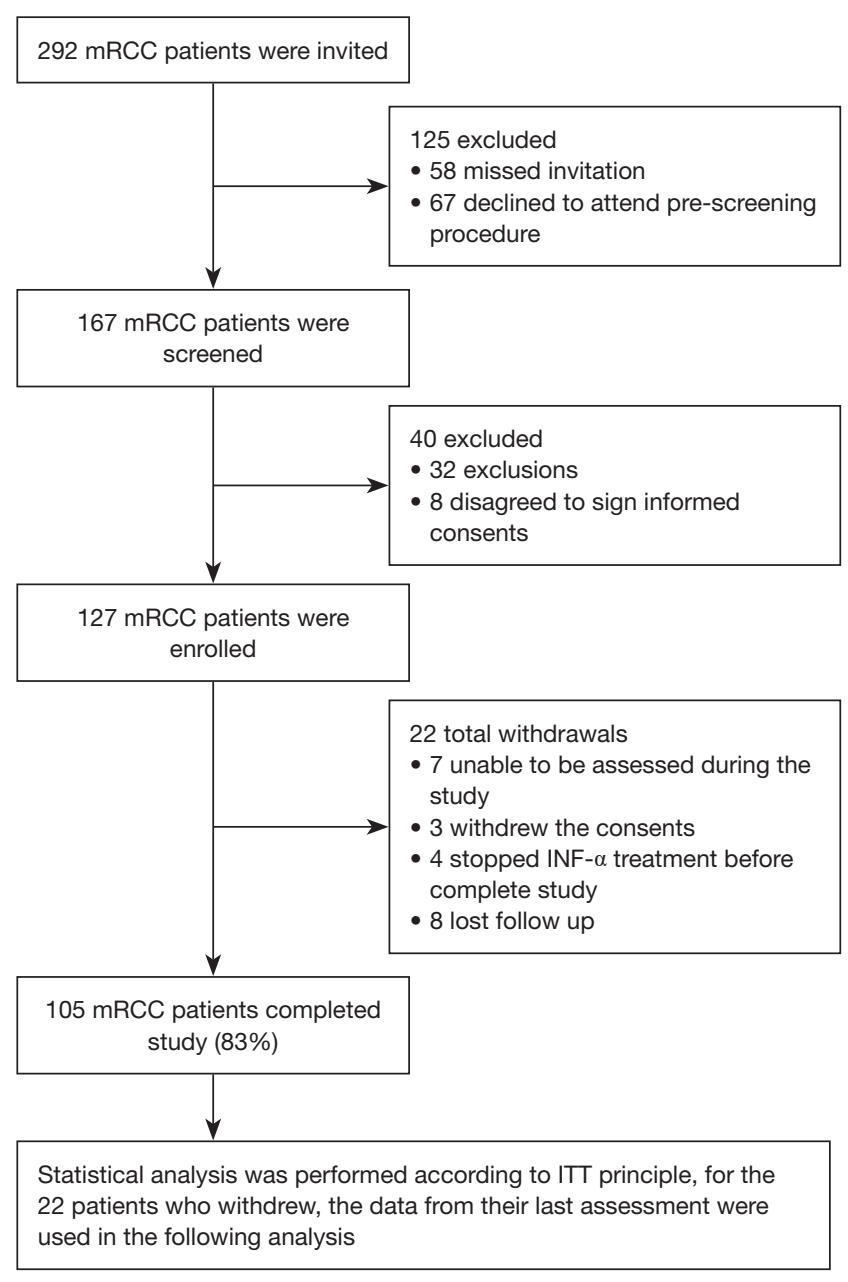

Figure 1 Study flow. MRCC, metastatic renal cell carcinoma.

anxiety/depression; 15-21, severe anxiety/depression (15).

\section{Assessments of quality of life}

We used European Organization for Research and Treatment of Cancer Quality of Life Questionnaire (EORTC QLQ-C30 Scale) to assess the QoL of patients at W0, W4, W8 and W12. The EORTC QLQ-C30 Scale is a 30 -item instrument with a four-point scale, from "not at all" to "very much", for items 1 to 28 ; and a seven-point scale for items 29 and 30. The QLQ-C30 could be divided into 15 domains including 5 functioning domains (physical functioning, role functioning, cognitive functioning, emotional functioning and social functioning), 3 symptoms domains (fatigue, pain, nausea and vomiting), 1 global health status/quality of life and 6 single items (each item as a domain). All raw data were transformed to a 0 to 100 point scale. Higher scores in the global health status scale and functional scale indicated better health state and functioning, while higher score in the symptom scale indicated excessive symptoms (16).

\section{Definitions}

According to the assessment criteria of the HADSAnxiety score, HADS-Depression score and EORTC QLQ-C30 Scale, the elevation of HADS-Anxiety score, HADS-Depression score and EORTC QLQ-C30 ScaleSymptoms score indicate aggravating anxiety, depression and symptoms, respectively. And the reduction of EORTC QLQ-C30 Scale-Global health status score as well as EORTC QLQ-C30 Scale-Functions score suggest loss of quality of life and functions, respectively. In order to evaluate the predictive factors of pejorative indexes, including HADS-Anxiety score, HADS-Depression score and EORTC QLQ-C30 Scale scores at W12, we defined the following changes of the indexes mentioned above as events: (I) the elevation of HADS-Anxiety score, HADSDepression score and EORTC QLQ-C30 Scale-Symptoms score at W12 compared to W0; (II) the reduction of EORTC QLQ-C30 Scale-Global health status score and EORTC QLQ-C30 Scale-Functions score at W12 compared to W0.

\section{Statistics}

Statistical analysis was performed using SPSS 22.0 software (IBM, Corp., Ltd, USA) and GraphPad Prism 6.0 software (GraphPad Software Inc., USA). All analyses were performed based on the intention-to-treat (ITT) principles with the last observation carried forward (LOCF) method from any of the three post-baseline measures. As for the 22 patients who withdrew, the data from their last assessment were used in the following analysis. Comparison between each visit was determined by paired t-test or $\mathrm{McNemar}$ test. Univariate and multivariate logistic regression analyses were performed to evaluate the factors affecting the aggravation of HADS score and EORTC QLQ-C30 Scale score. $\mathrm{P}<0.05$ was considered significant.

\section{Results}

\section{Study flow}

As shown in Figure 1, 292 mRCC patients were initially 
Table 1 Baseline characteristics of patients with mRCC

\begin{tabular}{|c|c|}
\hline Items & mRCC patients $(\mathrm{N}=127)$ \\
\hline Age (years) & $54.87 \pm 11.15$ \\
\hline Gender (male/female) & $101 / 26$ \\
\hline \multicolumn{2}{|l|}{ Highest education } \\
\hline Primary school or less & $68(53.5)$ \\
\hline High school & $40(31.5)$ \\
\hline Undergraduate or above & $19(15.0)$ \\
\hline BMI $\left(\mathrm{kg} / \mathrm{m}^{2}\right)$ & $24.71 \pm 2.61$ \\
\hline Overweight (BMI >24 kg/m²) & $75(59.1)$ \\
\hline Smoke & $49(38.6)$ \\
\hline Drink & $43(33.9)$ \\
\hline Hypertension & $87(68.5)$ \\
\hline Hyperlipidemia & $66(52.0)$ \\
\hline Diabetes & $18(14.2)$ \\
\hline \multicolumn{2}{|l|}{ ECOG performance } \\
\hline 0 & $96(75.6)$ \\
\hline 1 & $29(22.8)$ \\
\hline 2 & $2(1.6)$ \\
\hline \multicolumn{2}{|l|}{ Histology } \\
\hline Clear RCC & $103(81.1)$ \\
\hline Others & $24(18.9)$ \\
\hline \multicolumn{2}{|l|}{ Number of metastatic site } \\
\hline 1 & $120(94.5)$ \\
\hline$>1$ & $7(5.5)$ \\
\hline \multicolumn{2}{|l|}{ Location of metastatic site } \\
\hline Lung & $84(66.0)$ \\
\hline Liver & $11(8.7)$ \\
\hline Bone & $11(8.7)$ \\
\hline Others & $14(11.0)$ \\
\hline Lung and liver & $3(2.4)$ \\
\hline Lung and bone & $2(1.6)$ \\
\hline Lung and others & $1(0.8)$ \\
\hline Liver and bone & $1(0.8)$ \\
\hline Tumor size $>7 \mathrm{~cm}$ & $56(44.1)$ \\
\hline Prior radiotherapy & $4(3.1)$ \\
\hline Prior chemotherapy & $1(0.8)$ \\
\hline HADS-Anxiety score & $5.80 \pm 3.17$ \\
\hline $\begin{array}{l}\text { No anxiety (HADS-Anxiety score } \\
0-7)\end{array}$ & 99 (78.0) \\
\hline Anxiety (HADS-Anxiety score 8-21) & $28(22.0)$ \\
\hline \multicolumn{2}{|l|}{ HADS-Anxiety grade } \\
\hline Light (HADS-Anxiety score 8-10) & $18(14.1)$ \\
\hline $\begin{array}{l}\text { Moderate } \\
\text { (HADS-Anxiety score 11-14) }\end{array}$ & $8(6.3)$ \\
\hline
\end{tabular}

Table 1 (continued)
Table 1 (continued)

\begin{tabular}{lc}
\hline Items & mRCC patients (N=127) \\
\hline Severe (HADS-Anxiety score 15-21) & $2(1.6)$ \\
HADS-Depression score & $6.43 \pm 3.81$ \\
No depression & $87(68.5)$ \\
(HADS-Depression score 0-7) & \\
Depression & $40(31.5)$ \\
(HADS-Depression score 8-21) & \\
HADS-Depression grade & $23(18.1)$ \\
Light (HADS-Depression score 8-10) & $11(8.7)$ \\
Moderate & \\
(HADS-Depression score 11-14) & $6(4.7)$ \\
Severe & \\
(HADS-Depression score 15-21) & \\
EORTC QLQ-C30 Scale & $63.98 \pm 14.25$ \\
Global health status score & $69.42 \pm 18.59$ \\
Functions score & $29.83 \pm 15.45$ \\
Symptoms score
\end{tabular}

Data was presented as mean value \pm standard deviation or count (percentage). mRCC, metastatic renal cell carcinoma; BMI, body mass index; ECOG, Eastern Cooperative Oncology Group; HADS, Hospital Anxiety and Depression Scale; EORTC QLQ-C30 Scale, European Organization for Research and Treatment of Cancer Quality of Life Questionnaire.

invited and 125 patients were excluded due to that there were 58 patients missed invitation and 67 patients declined to attend pre-screening procedure. Afterwards, $167 \mathrm{mRCC}$ patients were screened for eligibility and 40 patients were excluded (32 exclusions and 8 disagreed to sign informed consents), so the remaining $127 \mathrm{mRCC}$ patients were enrolled in our study. Among the $127 \mathrm{mRCC}$ patients, there were 22 withdrawals including 7 patients unable to be assessed during the study, 3 patients withdrew the consents, 4 patients stopped IFN- $\alpha$ treatment before complete study and 8 patients lost to follow up, thus leaving $105 \mathrm{mRCC}$ patients completed the whole study which accounted for $83 \%$ of our cohort. As for the 22 withdrew patients, the data collected from their last assessments were used in the following analysis. The statistical analysis was performed according to the ITT principles using the LOCF method.

\section{Baseline characteristics}

The mean age of the total $127 \mathrm{mRCC}$ patients was $54.87 \pm 11.15$ years, among whom there were 101 males and 26 females (Table 1). And the numbers of patients had the 
A

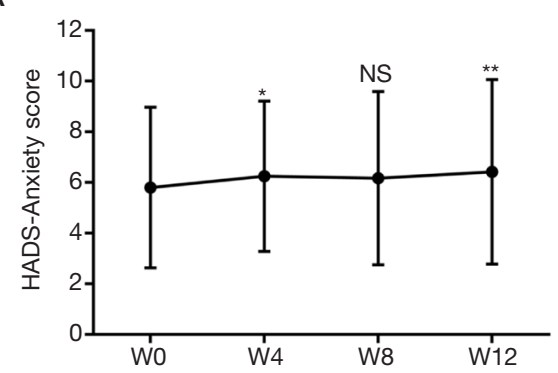

B

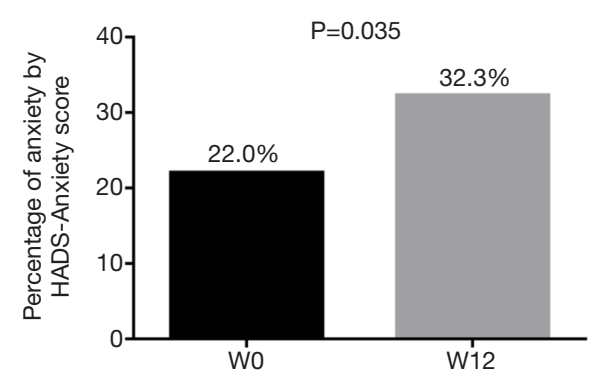

Figure 2 Change of HADS-Anxiety score. HADS-Anxiety score was higher at W4 and W12 compared with W0, while no difference was observed between W8 and W0 (A), and the percentage of patients had anxiety evaluated by HADS-Anxiety score was increased at W12 compared with W0 (B). Comparison between two groups was determined by paired $t$ test. $\mathrm{P}<0.05$ was considered significant. HADS, Hospital Anxiety and Depression Scale. ${ }^{*} \mathrm{P}<0.05 ;{ }^{* *} \mathrm{P}<0.01 ; \mathrm{NS}$, not significant.

A

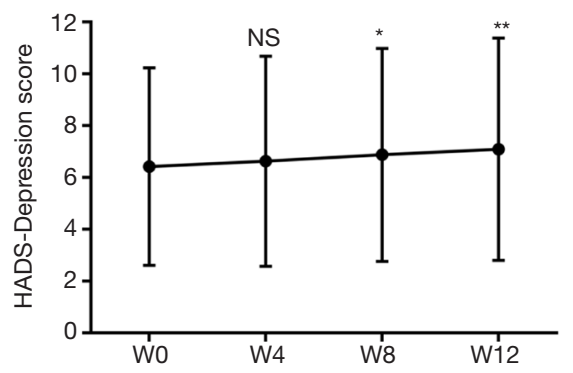

B

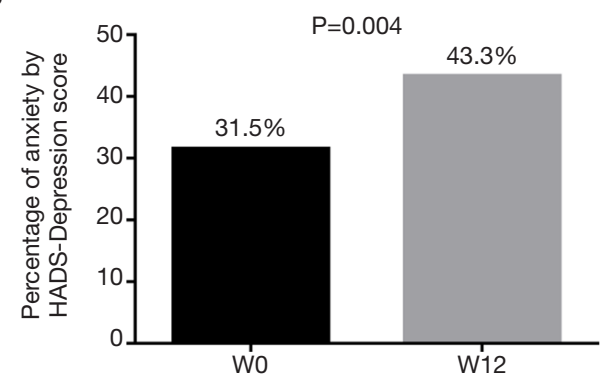

Figure 3 Change of HADS-Depression score. HADS-Depression score increased at W8 and W12, however, it was of no difference at W4 compared with W0 (A), and the percentage of patients had depression assessed by HADS-Depression score was elevated at W12 compared with W0 (B). Comparison between two groups was determined by paired $t$ test. $\mathrm{P}<0.05$ was considered significant. HADS, Hospital Anxiety and Depression Scale. ${ }^{*} \mathrm{P}<0.05 ;{ }^{*} \mathrm{P}<0.01$; NS, not significant.

highest education of primary school or less, high school and undergraduate or above were 68 (53.5\%), 40 (31.5\%) and 19 (15.0\%), respectively. The mean value of BMI and the number of overweight patients were $24.71 \pm 2.61 \mathrm{~kg} / \mathrm{m}^{2}$ and $75(59.1 \%)$, respectively. And the number of patients had ECOG performance score of 0,1 and 2 were 96 (75.6\%), $29(22.8 \%)$ and $2(1.6 \%)$, respectively. In addition, there were $103(81.1 \%)$ patients had clear RCC and 24 (18.9\%) patients had other RCCs. And the numbers of patients had 1 and $>1$ metastatic site were 120 (94.5\%) and $7(5.5 \%)$, respectively. The other information of medical history, clinicopathological features, HADS and EORTC QLQ-C30 Scale scores at baseline were listed in Table 1.

\section{Change of HADS-Anxiety score and HADS-depression score}

As presented in Figure 2, the HADS-Anxiety score increased at W4 $(\mathrm{P}<0.05)$ and $\mathrm{W} 12(\mathrm{P}<0.01)$ compared with $\mathrm{W} 0$, while no difference was observed between $\mathrm{W} 8$ and $\mathrm{W} 0$ $(\mathrm{P}>0.05)$ (Figure 2A). At W12, the percentage of patients had anxiety assessed by HADS-Anxiety score was elevated compared with W0 (32.3\% vs. 22.0\%, $\mathrm{P}=0.035)$ (Figure 2B). The HADS-Depression score was higher at W8 $(\mathrm{P}<0.05)$ and $\mathrm{W} 12(\mathrm{P}<0.01)$ than that of $\mathrm{W} 0$, however, the HADSDepression score at $\mathrm{W} 4$ was of no difference compared with W0 (Figure $3 A$ ). And the proportion of patients had depression assessed by HADS-Depression score was increased at W12 than that of W0 (43.3\% vs. $31.5 \%$, $\mathrm{P}=0.004$ ) (Figure 3B).

\section{Change of EORTC QLQ-C30 Scale scores}

The EORTC QLQ-C30 Scale-Global health status score did not change at W4, W8 or W12 compared to W0 (all 
A

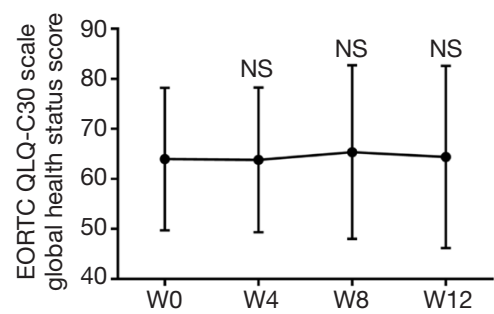

B

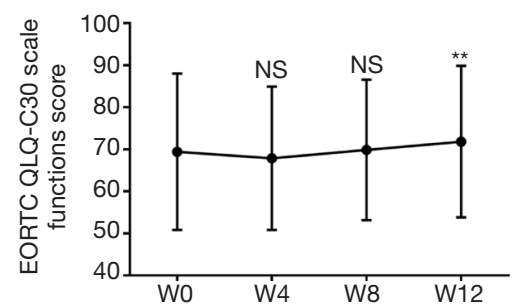

C

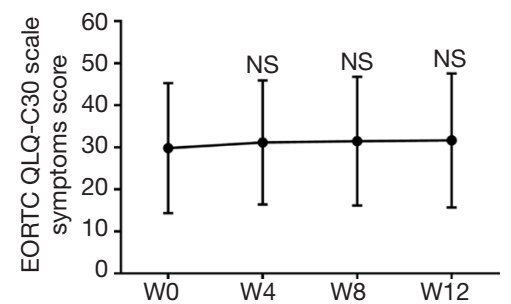

Figure 4 Change of EORTC QLQ-C30 Scale scores. The EORTC QLQ-C30 Scale-Global health status score (A) and EORTC QLQ-C30 Scale-Symptoms scores (C) did not vary at W4, W8 or W12 compared to W0. The EORTC QLQ-C30 Scale-Function score increased at W12 while was similar to $\mathrm{W} 0$ at $\mathrm{W} 4$ and $\mathrm{W} 8$ (B). Comparison between two groups was determined by paired $\mathrm{t}$ test. $\mathrm{P}<0.05$ was considered significant. EORTC QLQ, European Organization for Research and Treatment of Cancer Quality of Life Questionnaire. ${ }^{* *} \mathrm{P}<0.01$; NS, not significant.

Table 2 Number of patients with changed HADS-Anxiety, HADSDepression and EORTC QLQ-C30 Scale scores from week 0 (W0) to week $12(\mathrm{~W} 12)$

\begin{tabular}{|c|c|}
\hline Scores & Patients \\
\hline \multicolumn{2}{|c|}{ HADS-Anxiety score } \\
\hline Elevated & $64(50.4)$ \\
\hline Reduced & $36(28.3)$ \\
\hline Unchanged & $27(21.3)$ \\
\hline \multicolumn{2}{|c|}{ HADS-Depression score } \\
\hline Elevated & $62(48.8)$ \\
\hline Reduced & $34(26.8)$ \\
\hline Unchanged & $31(24.4)$ \\
\hline \multicolumn{2}{|c|}{ EORTC QLQ-C30 Scale-Global health status score } \\
\hline Elevated & $52(40.9)$ \\
\hline Reduced & $51(40.2)$ \\
\hline Unchanged & $24(18.9)$ \\
\hline \multicolumn{2}{|c|}{ EORTC QLQ-C30 Scale-Functions score } \\
\hline Elevated & $67(52.8)$ \\
\hline Reduced & $52(40.9)$ \\
\hline Unchanged & $8(6.3)$ \\
\hline \multicolumn{2}{|c|}{ EORTC QLQ-C30 Scale-Symptoms score } \\
\hline Elevated & $67(52.8)$ \\
\hline Reduced & $57(44.9)$ \\
\hline Unchanged & $3(2.3)$ \\
\hline
\end{tabular}

Data was presented as count (percentage). HADS, Hospital Anxiety and Depression Scale; EORTC QLQ-C30 Scale, European Organization for Research and Treatment of Cancer Quality of Life Questionnaire.
$\mathrm{P}>0.05$ ) (Figure 4A). As to EORTC QLQ-C30 ScaleFunction score, it increased at W12 $(\mathrm{P}<0.01)$ compared to $\mathrm{W} 0$, while was similar to $\mathrm{W} 0$ at $\mathrm{W} 4(\mathrm{P}>0.05)$ and $\mathrm{W} 8$ $(\mathrm{P}>0.05)$ (Figure $4 B)$. As shown in Figure $4 C$, the EORTC QLQ-C30 Scale-Symptoms scores at W4 (P>0.05), W8 $(\mathrm{P}>0.05)$ and $\mathrm{W} 12(\mathrm{P}>0.05)$ were similar to that of $\mathrm{W} 0$.

\section{Change of HADS scores and EORTC QLQ-C30 Scale scores from W12 to WO}

The numbers of patients had elevated, reduced and unchanged HADS scores and EORTC QLQ-C30 scores at W12 compared with W0 were displayed in Table 2. Sixty-four (50.4\%), $36(28.3 \%)$ and $27(21.3 \%)$ patients had elevated, reduced and unchanged HADS-Anxiety score, and the numbers of patients with elevated, reduced and unchanged HADS-Depression score were 62 (48.8\%), $34(26.8 \%)$ and $31(24.4 \%)$, respectively. In addition, the numbers of patients had elevated, reduced and unchanged EORTC QLQ-C30 Scale-Global health status score were 52 (40.9\%), 51 (40.2\%) and 24 (18.9\%), respectively. As for ORTC QLQ-C30 Scale-Function score, it was elevated in $67(52.8 \%)$ patients, reduced in $52(40.9 \%)$ patients and unchanged in $8(6.3 \%)$ patients. And the figures of patients with elevated, reduced and unchanged ORTC QLQ-C30 Scale-Symptoms score were 67 (52.8\%), 57 (44.9\%) and $3(2.3 \%)$, respectively.

\section{Factors affecting the aggravation of HADS scores at W12}

In order to observe the predictive factors for the aggravation of HADS scores, univariate and multivariate logistic regression analyses were conducted. As displayed in Table 3, 
Table 3 Factors affecting the elevation of HADS-Anxiety score at week 12 (W12) compared to week 0 (W0) by logistic regression model analysis

\begin{tabular}{|c|c|c|c|c|c|c|}
\hline Parameters & \multicolumn{3}{|c|}{ Univariate logistic regression } & \multicolumn{3}{|c|}{ Multivariate logistic regression } \\
\hline Age & 0.006 & 0.952 & $0.919-0.986$ & $<0.001$ & 0.878 & $0.822-0.938$ \\
\hline Gender (male) & 0.029 & 0.428 & $0.200-0.918$ & 0.356 & 0.637 & $0.244-1.659$ \\
\hline Higher highest education & 0.298 & 0.775 & $0.480-1.252$ & 0.005 & 0.314 & $0.140-0.705$ \\
\hline Overweight & 0.010 & 2.605 & $1.256-5.403$ & 0.343 & 2.019 & $0.473-8.612$ \\
\hline Smoke & 0.401 & 1.360 & $0.664-2.786$ & 0.686 & 1.202 & $0.493-2.931$ \\
\hline Drink & 0.901 & 1.048 & $0.502-2.185$ & 0.707 & 1.198 & $0.467-3.071$ \\
\hline Hypertension & 0.007 & 2.942 & $1.339-6.465$ & 0.009 & 3.903 & $1.414-10.774$ \\
\hline Higher ECOG performance & 0.376 & 1.401 & $0.664-2.953$ & 0.402 & 1.541 & $0.560-4.237$ \\
\hline Histology (clear RCC vs. others) & 0.344 & 1.543 & $0.628-3.791$ & 0.364 & 1.705 & $0.539-5.392$ \\
\hline Number of metastatic site (>1 vs. 1 ) & 0.714 & 1.333 & $0.286-6.214$ & 0.325 & 2.524 & $0.400-15.947$ \\
\hline Metastatic site (lung vs. others) & 0.521 & 1.286 & $0.597-2.770$ & 0.632 & 1.285 & $0.461-3.581$ \\
\hline Tumor size $>7 \mathrm{~cm}$ & 0.781 & 1.105 & $0.548-2.227$ & 0.959 & 1.024 & $0.412-2.547$ \\
\hline Prior radiotherapy & 0.340 & 3.049 & $0.309-30.129$ & 0.082 & 18.059 & $0.689-473.357$ \\
\hline Prior chemotherapy & 1.000 & - & 0.000 & 1.000 & - & 0.000 \\
\hline
\end{tabular}

Data was presented as $\mathrm{P}$ value, OR (odds ratio) and $95 \% \mathrm{Cl}$ (confidence interval). Factors affecting the elevation of HADS-Anxiety score at week 12 (W12) compared to week 0 (W0) were determined by univariate and multivariate logistic regression model analysis. Highest education was scored as: primary school or less $=0$, high school $=1$ and undergraduate or above $=2$. $P$ value $<0.05$ was considered significant. HADS, Hospital Anxiety and Depression Scale; BMI, body mass index; ECOG, Eastern Cooperative Oncology Group.

univariate logistic regression analysis revealed that older age $(\mathrm{P}=0.006)$ and gender male $(\mathrm{P}=0.029)$ correlated with less risk of elevated HADS-Anxiety score at W12, while overweight $(\mathrm{P}=0.010)$ and hypertension $(\mathrm{P}=0.007)$ associated with higher risk of increased HADS-Anxiety score at W12. All factors were included in the multivariate logistic regression analysis, which disclosed that older age $(\mathrm{P}<0.001)$ and elevated level of highest education $(\mathrm{P}=0.005)$ independently predict reduced risk of HADS-Anxiety score elevation at $\mathrm{W} 12$, while hypertension $(\mathrm{P}=0.009)$ was an independent predictive factor for increased risk.

As for the predicting factors for elevated HADSDepression score, univariate logistic regression analysis disclosed that age $(\mathrm{P}=0.014)$, gender male $(\mathrm{P}=0.005)$ and metastatic site at lung $(\mathrm{P}=0.022)$ associated with limited risk of elevated HADS-Depression score (Table 4). All factors were included in the multivariate logistic regression analysis, which showed that age $(\mathrm{P}=0.005)$ independently associated with less risk of elevated HADS-Depression score.

\section{Factors affecting the aggravation of EORTC QLQ-C30 Scale scores at W12}

In order to assess the predictive factors for the aggravation of EORTC QLQ-C30 Scale scores (the reductions of EORTC QLQ-C30 Scale-Global health status score and EORTC QLQ-C30 Scale-Functions score, and the elevation of EORTC QLQ-C30 Scale-Symptoms score), univariate and multivariate logistic regression analyses were performed. As exhibited in Table 5 , gender male $(\mathrm{P}=0.050)$ and metastatic site at lung $(\mathrm{P}=0.043)$ correlated with decreased risk of EORTC QLQ-C30 Scale-Global health status score reduction at $\mathrm{W} 12$, however, smoke $(\mathrm{P}=0.020)$, 
Table 4 Factors affecting the elevation of HADS-Depression score at week 12 (W12) compared to week 0 (W0) by logistic regression model analysis

\begin{tabular}{|c|c|c|c|c|c|c|}
\hline Parameters & \multicolumn{3}{|c|}{ Univariate logistic regression } & \multicolumn{3}{|c|}{ Multivariate logistic regression } \\
\hline Age & 0.014 & 0.958 & $0.926-0.991$ & 0.005 & 0.933 & $0.889-0.980$ \\
\hline Gender (male) & 0.005 & 0.333 & $0.154-0.723$ & 0.085 & 0.451 & $0.182-1.117$ \\
\hline Higher highest education & 0.615 & 0.885 & $0.549-1.425$ & 0.073 & 0.542 & $0.278-1.058$ \\
\hline Overweight & 0.889 & 1.052 & $0.518-2.134$ & 0.095 & 3.439 & $0.806-14.681$ \\
\hline Smoke & 0.449 & 1.319 & $0.644-2.699$ & 0.918 & 0.956 & $0.407-2.244$ \\
\hline Drink & 0.705 & 1.152 & $0.552-2.404$ & 0.838 & 0.911 & $0.374-2.218$ \\
\hline Hypertension & 0.335 & 1.450 & $0.681-3.084$ & 0.383 & 1.490 & $0.608-3.649$ \\
\hline Higher ECOG performance & 0.281 & 1.508 & $0.714-3.185$ & 0.733 & 1.171 & $0.473-2.897$ \\
\hline Histology (clear RCC vs. others) & 0.561 & 0.768 & $0.315-1.872$ & 0.129 & 0.437 & $0.150-1.274$ \\
\hline Number of metastatic site (>1 vs. 1 ) & 0.235 & 2.763 & $0.516-14.804$ & 0.069 & 5.919 & $0.873-40.144$ \\
\hline Metastatic site (lung vs. others) & 0.022 & 0.396 & $0.179-0.876$ & 0.149 & 0.493 & $0.188-1.287$ \\
\hline Tumor size $>7 \mathrm{~cm}$ & 0.813 & 1.088 & $0.540-2.193$ & 0.717 & 1.169 & $0.501-2.727$ \\
\hline Prior radiotherapy & 0.354 & 0.339 & $0.034-3.348$ & 0.192 & 0.184 & $0.015-2.337$ \\
\hline Prior chemotherapy & 1.000 & - & 0.000 & 1.000 & - & 0.000 \\
\hline
\end{tabular}

Data was presented as $\mathrm{P}$ value, OR (odds ratio) and 95\% Cl (confidence interval). Factors affecting the elevation of HADS-Depression score at week 12 (W12) compared to week 0 (W0) were determined by univariate and multivariate logistic regression model analysis. Highest education was scored as: primary school or less $=0$, high school $=1$ and undergraduate or above $=2$. $P$ value $<0.05$ was considered significant. HADS, Hospital Anxiety and Depression Scale; BMI, body mass index; ECOG, Eastern Cooperative Oncology Group.

hypertension $(\mathrm{P}=0.007)$, diabetes $(\mathrm{P}=0.018)$ and higher ECOG performance status $(\mathrm{P}=0.005)$ correlated with higher risk. And multivariate logistic regression analysis revealed that smoke $(\mathrm{P}=0.037)$, hypertension $(\mathrm{P}=0.003)$, diabetes $(\mathrm{P}=0.011)$ and higher ECOG performance status $(\mathrm{P}=0.004)$ were independent predicting factors for escalated risk of EORTC QLQ-C30 Scale-Global health status score reduction at $\mathrm{W} 12$.

As for the predictive factors for risk of the reduced EORTC QLQ-C30 Scale-Function score at W12, univariate logistic regression analysis showed that higher ECOG performance status $(\mathrm{P}=0.016)$ correlated with increased risk of reduced EORTC QLQ-C30 ScaleFunction score at W12 (Table 6). Subsequently, all factors with a $\mathrm{P}$ value $<0.1$ were analyzed by the multivariate logistic regression analysis, which disclosed that higher ECOG performance status $(\mathrm{P}=0.026)$ was an independent predicting factor for increased risk of reduction of EORTC QLQ-C30 Scale-Function score at W12.

As to the EORTC QLQ-C30 Scale-Symptoms score, univariate and multivariate logistic regression analyses were performed to assess the predictive factors for its aggravation as well. As presented in Table 7, the univariate logistic regression analysis displayed that overweight $(\mathrm{P}=0.021)$ correlated with increased risk of EORTC QLQ-C30 Scale-Symptoms score increment at W12. All factors were included in the multivariate logistic regression analysis, while no factor was revealed as an independent predictive factor for elevated EORTC QLQ-C30 Scale-Symptoms score at W12. 
Table 5 Factors affecting the reduction of EORTC QLQ-C30 Scale-Global health status score at week 12 (W12) compared to week 0 (W0) by logistic regression model analysis

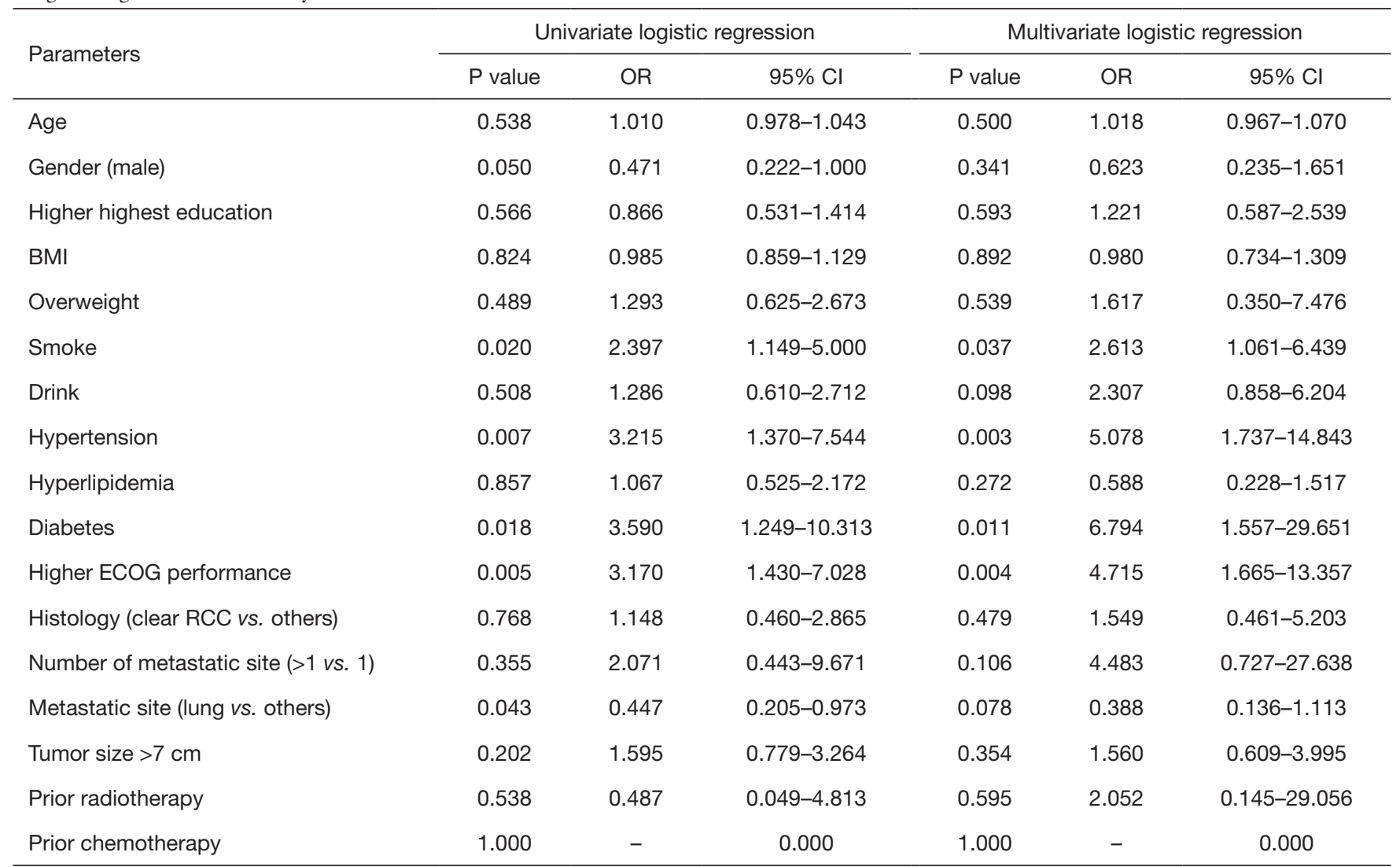

Data was presented as $\mathrm{P}$ value, OR (odds ratio) and 95\% Cl (confidence interval). Factors affecting the reduction of EORTC QLQ-C30 Scale-Global health status score at week 12 (W12) compared to week 0 (W0) were determined by univariate and multivariate logistic regression model analysis. Highest education was scored as: primary school or less $=0$, high school $=1$ and undergraduate or above $=2$.

$P$ value $<0.05$ was considered significant. EORTC QLQ-C30 Scale, European Organization for Research and Treatment of Cancer Quality of Life Questionnaire; BMI, body mass index; ECOG, Eastern Cooperative Oncology Group.

\section{Discussion}

In our study, we found that: (I) in mRCC patients treated by IFN- $\alpha$, the HADS-Anxiety score was elevated at W4 and W12, HADS-Depression score was higher at W8 and W12 compared with W0, and the anxiety and depression percentages were both increased at W12 than that of W0. In addition, the EORTC QLQ-Global health status score, EORTC QLQ-Symptoms score and ORTC QLQFunction score did not vary at W4, W8 or W12 compared with W0; (II) multivariate logistic regression disclosed that older age and elevated level of highest education were independent predictive factors for reduced risk of HADS-Anxiety score elevation, while hypertension was independently associated with increased risk. Additionally, older age was an independent predictive factor for decreased risk of HADS-Depression score elevation; (III) as for EORTC QLQ scores, the multivariate logistic regression illuminated that smoke, hypertension, diabetes and higher ECOG performance independently predicted increased risk of reduced EORTC QLQ-Global health score, and higher ECOG performance was also an independent predictive factor for EORTC QLQ-Function score reduction, while no factor independently correlated with the aggravation of EORTC QLQ-C30 Scale-Symptoms score at W12.

As a grievous condition and a major threat to human, cancer directly or indirectly causes mood disorders to patients as a consequence of a large reduction of life expectancy, side effect of anti-tumor drugs and a change of quality of life $(11,17,18)$. For instance, in a recent cohort study in Indonesia, the researchers discover an obvious decline in anxiety level and an increase of QoL 
Table 6 Factors affecting the reduction of EORTC QLQ-C30 Scale-Functions score at week 12 (W12) compared to week 0 (W0) by logistic regression model analysis

\begin{tabular}{|c|c|c|c|c|c|c|}
\hline Parameters & \multicolumn{3}{|c|}{ Univariate logistic regression } & \multicolumn{3}{|c|}{ Multivariate logistic regression } \\
\hline Age & 0.364 & 0.985 & $0.954-1.017$ & 0.528 & 0.986 & $0.945-1.029$ \\
\hline Gender (male) & 0.758 & 0.889 & $0.420-1.881$ & 0.675 & 1.208 & $0.498-2.930$ \\
\hline Higher highest education & 0.451 & 1.203 & $0.744-1.947$ & 0.676 & 1.142 & $0.613-2.130$ \\
\hline Overweight & 0.915 & 1.040 & $0.506-2.136$ & 0.964 & 0.969 & $0.252-3.728$ \\
\hline Smoke & 0.257 & 0.653 & $0.312-1.366$ & 0.073 & 0.457 & $0.194-1.074$ \\
\hline Drink & 0.321 & 0.681 & $0.318-1.456$ & 0.439 & 0.714 & $0.303-1.679$ \\
\hline Hypertension & 0.593 & 1.233 & $0.572-2.659$ & 0.726 & 1.166 & $0.493-2.760$ \\
\hline Higher ECOG performance & 0.016 & 2.587 & $1.190-5.624$ & 0.026 & 2.731 & $1.126-6.624$ \\
\hline Histology (clear RCC vs. others) & 0.703 & 1.194 & $0.479-2.981$ & 0.873 & 0.918 & $0.325-2.598$ \\
\hline Number of metastatic site (>1 vs. 1 ) & 0.916 & 1.087 & $0.233-5.072$ & 0.984 & 1.018 & $0.175-5.927$ \\
\hline Metastatic site (lung vs. others) & 0.736 & 0.875 & $0.403-1.900$ & 0.942 & 1.035 & $0.414-2.589$ \\
\hline Tumor size $>7 \mathrm{~cm}$ & 0.067 & 1.958 & $0.955-4.017$ & 0.137 & 1.867 & $0.820-4.249$ \\
\hline Prior radiotherapy & 0.710 & 1.460 & $0.199-10.710$ & 0.675 & 1.611 & $0.173-14.997$ \\
\hline Prior chemotherapy & 1.000 & 0.000 & 0.000 & 1.000 & 0.000 & 0.000 \\
\hline
\end{tabular}

Data was presented as $\mathrm{P}$ value, OR (odds ratio) and 95\% Cl (confidence interval). Factors affecting the reduction of EORTC QLQ-C30 Scale-Functions score at week 12 (W12) compared to week 0 (W0) were determined by univariate and multivariate logistic regression model analysis. Highest education was scored as: primary school or less $=0$, high school $=1$ and undergraduate or above $=2$.

$P$ value $<0.05$ was considered significant. EORTC QLQ-C30 Scale, European Organization for Research and Treatment of Cancer Quality of Life Questionnaire; BMI, body mass index; ECOG, Eastern Cooperative Oncology Group.

in oral squamous cell carcinoma patients treated by chemotherapy or invasive therapies (19). Despite that metaanalysis demonstrate encouraging treatment responses and reduced mortality rate in mRCC patients treated by IFN- $\alpha$ compared with placebo or non-immunosuppressive therapies, anxiety and depression are still frequently observed in clinical practice and clinical studies $(20,21)$. Nonetheless, studies investigating the incidences of anxiety and depression of mRCC patients treated by IFN- $\alpha$ are rare, most of those studies are conducted on patients with hepatitis. In a prospective cohort study enrolls patients with hepatitis $\mathrm{C}$ treated by pegylated interferon (PEG-IFN)based treatment, they find that there are $10.4 \%$ patients developing major depressive disorder and $11.9 \%$ patients are diagnosed as subsyndromal depression (22). Another retrospective cohort study evaluates the psychiatric status of patients with hepatitis $\mathrm{C}$ treated by IFN- $\alpha$ and patients who stop the IFN- $\alpha$ treatment, their results exhibit that the incidence of depression and anxiety are notably elevated in patients treated by IFN- $\alpha$ compared with those stopping the IFN- $\alpha$ treatment (23). In our study, at W12 after the IFN- $\alpha$ treatment, we found that the HADS-Anxiety score and HADS-Depression score both increased after treatment and the percentages of patients had depression and anxiety evaluated by HADS were also elevated, which could be explained as follows: as a pro-inflammatory cytokine, IFN- $\alpha$ is illuminated to be able to lower the level of tryptophan (TRP), which is a precursor of serotonin, through inducing the TRP to transfer to kynurenine (KYN) (24-27). QoL is another aspect in the management of mRCC patients treated by IFN- $\alpha$, however is not regularly assessed by clinical studies or trials. In a cohort study enrolls $21 \mathrm{mRCC}$ 
Table 7 Factors affecting the elevation of EORTC QLQ-C30 Scale-Symptoms score at week 12 (W12) compared to week 0 (W0) by logistic regression model analysis

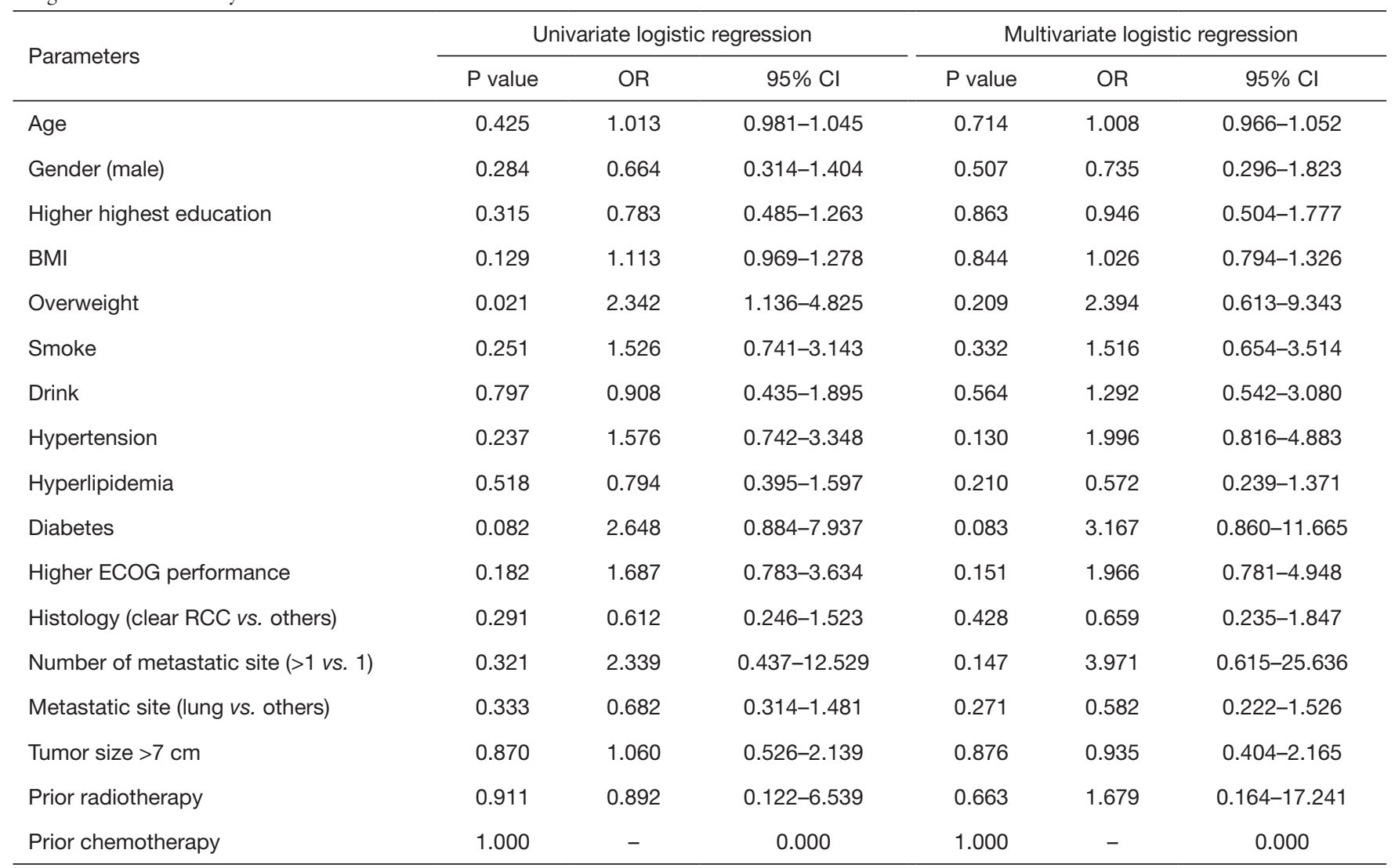

Data was presented as $\mathrm{P}$ value, OR (odds ratio) and 95\% Cl (confidence interval). Factors affecting the elevation of EORTC QLQ-C30 Scale-Symptoms score at week 12 (W12) compared to week 0 (W0) were determined by univariate and multivariate logistic regression model analysis. Highest education was scored as: primary school or less $=0$, high school $=1$ and undergraduate or above $=2$.

$P$ value $<0.05$ was considered significant. EORTC QLQ-C30 Scale, European Organization for Research and Treatment of Cancer Quality of Life Questionnaire; BMI, body mass index; ECOG, Eastern Cooperative Oncology Group.

patients treated by the combination of erythropoietin, IFN- $22 \mathrm{a}$ and interleukin (IL)-2, their results showed that the QoL assessed by Functional Assessment of Cancer Therapy scale of 10 patients is elevated after treatment (28). In our study, the EORTC QLQ-Global health status score, EORTC QLQ-Symptoms score and EORTC QLQFunction score did not deteriorate throughout the whole course of treatment, indicating the QoL of mRCC patients in our study treated by IFN- $\alpha$ did not aggravated after treatment. This result in our study might be due to that the burden of disease relieved after treatment, and the EORTC QLQ-Function score was elevated at W12 and the EORTC QLQ-Symptoms score did not vary throughout the whole treatment course, which contributes to the unchanged score of EORTC QLQ-Global health status.

Despite that depression and anxiety of patients with
mRCC treated by IFN- $\alpha$ are occasionally reported, the effort of evaluating the predicting factor of this issue is still scarce. And in view that there might be difference of anxiety and depression in mRCC patients treated by IFN- $\alpha$, exploring the predicting factors for the incidences of anxiety and depression might provide guidance for the patient selection in clinical practice. To our best knowledge, this is the first study to investigate predictive factors for the incidence of depression and anxiety in mRCC patients treated by IFN- $\alpha$, and the majority of prior studies only focus on patients with hepatitis and other solid tumors (29-31). A prospective cohort study illustrates that severity of preexisting depressive symptoms and additional physical comorbidities predict early onset of depression, and additional physical comorbidities as well as depressive temperament associate with persistent depression in 
hepatitis C (HCV) patients treated by IFN- $\alpha$ (29). And another cohort study elucidates that social support decreases the depression induced by low-dose IFN- $\alpha$ treatment in melanoma patients (31). The studies investigating the predictive value of baseline characteristics for depression and anxiety in cancer patients receiving IFN- $\alpha$ treatment are very rare. An earlier study conducted by Capuron et al. illustrates that RCC or malignant melanoma patients with higher Montgomery-Asberg depression rating scale (MADRS) scores at baseline present with moderate to severe depressive symptoms after IL-2 and/or IFNalpha treatment, and baseline emotional symptoms, sleep disorders and low social support are predictors for depressive symptoms at the first month of IL-2 and/or IFNalpha treatment (32). However, in their study, the sample size was small $(\mathrm{N}=32)$, and they included not only RCC patients but also malignant melanoma patients. In our study, older age, elevated level of highest education independently associated with less risk of HADS-Anxiety score elevation post treatment, while hypertension independently correlated with increased risk. As for depression, older age was also an independent predicting factor for reduced risk of increased HADS-Depression score. Those results in our study could be explained as follows: (I) older adults have stronger expectancy for a positive future compared with younger adults when they both have initial negative or ambiguous scenario, indicating the elderly might be less likely to develop depression and anxiety (33); (II) a higher education level is related to an obvious increase of selfassessed health level, life satisfaction and quality of life, which suggests a higher education level may reduce the risk of anxiety (34); (III) as to hypertension, it is elucidated to be highly correlated with symptoms of depression (35).

Accumulating studies confirm that QoL is another aspect crucial to cancer patients treated by IFN- $\alpha$. A multicenter, open-label, prospective randomized phase III trial reports that the QoL are the same between resected melanoma patients treated by high dose IFN- $\alpha-2 \mathrm{a}$ and low dose IFN$\alpha-2 a$ (36). Nonetheless, there is still no study evaluating the predictive factors for QoL in mRCC patients treated by IFN- $\alpha$, and the studies investigating the predicting value of baseline characteristics for the change of QoL in patients with other cancers treated by IFN- $\alpha$ are few, either. A study that is conducted earlier in 2002 elucidates that the toxicity of IFN- $\alpha-2 b$ correlates with the change of QoL score in melanoma patients (37). In our study, the QoL was assessed by the EORTC QLQ-C30 Scale, and the multivariate logistic regressions revealed that smoke, hypertension, diabetes and higher ECOG performance independently predict higher risk of EORTC QLQ-Global health status score reduction, and higher ECOG performance was also an independent predictive factor for reduced EORTC QLQ-Function score. In addition, no baseline characteristic was found to be correlated with the escalation of EORTC QLQ-Symptoms score. There are several probable explanations for our results: (I) smoking pejoratively affects the treatment response, survival and performance status in patients with various cancers, indicating smoking may decrease the QoL in patients by impairing the efficacy of treatments $(38,39)$; (II) as to diabetes and hypertension, the symptoms such as dizziness, headache and fatigue caused by hypertension, and eating disorder, fatigue as well as emaciation resulted from diabetes, could notably decrease the QoL of cancer patients; (III) ECOG performance status illustrates the physical status of cancer patients aims to evaluate their tolerance to multiple treatments, a higher ECOG performance status suggests a more unsatisfactory physical condition and less tolerance to the treatments, which could result in a poorer QoL.

There were several limitations in this study: (I) our study was conducted in a single center that might result in a selection bias in our study; (II) the sample size was small; (III) the long-term evaluation of anxiety, depression and QoL in mRCC patients treated by IFN- $\alpha$ in our study were not assessed.

In conclusion, anxiety and depression increased while QoL did not deteriorate in mRCC patients during IFN- $\alpha$ treatment, and age, highest education, hypertension, smoke, diabetes as well as higher ECOG performance status correlated with their aggravation.

\section{Acknowledgments}

Funding: None.

\section{Footnote}

Conflicts of Interest: All authors have completed the ICMJE uniform disclosure form (available at http://dx.doi. org/10.21037/tcr.2018.11.07). The authors have no conflicts of interest to declare.

Ethical Statement: The authors are accountable for all aspects of the work in ensuring that questions related to the accuracy or integrity of any part of the work are appropriately investigated and resolved. The study was conducted in 
accordance with the Declaration of Helsinki (as revised in 2013). This study was approved by the Ethics Committee of Harbin Medical University Cancer Hospital and conducted according to the Helsinki Declaration. The number of the Ethic Approval was KY2012-12. All patients provided written informed consents.

Open Access Statement: This is an Open Access article distributed in accordance with the Creative Commons Attribution-NonCommercial-NoDerivs 4.0 International License (CC BY-NC-ND 4.0), which permits the noncommercial replication and distribution of the article with the strict proviso that no changes or edits are made and the original work is properly cited (including links to both the formal publication through the relevant DOI and the license). See: https://creativecommons.org/licenses/by-nc-nd/4.0/.

\section{References}

1. Chow WH, Dong LM, Devesa SS. Epidemiology and risk factors for kidney cancer. Nat Rev Urol 2010;7:245-57.

2. Torre LA, Bray F, Siegel RL, et al. Global cancer statistics, 2012. CA Cancer J Clin 2015;65:87-108.

3. Ferlay J, Soerjomataram I, Ervik M, et al. GLOBOCAN 2012 v1.0, Cancer Incidence and Mortality Worldwide: IARC CancerBase No. 11. Lyon, France: International Agency for Research on Cancer, Available online: http:// globocan.iarc.fr/. 2013.

4. Chen $\mathrm{W}$, Zheng R, Baade PD, et al. Cancer statistics in China, 2015. CA Cancer J Clin 2016;66:115-32.

5. Rini BI, Campbell SC, Escudier B. Renal cell carcinoma. Lancet 2009;373:1119-32.

6. Kawano Y, Takahashi W, Eto M, et al. Prognosis of metastatic renal cell carcinoma with first-line interferonalpha therapy in the era of molecular-targeted therapy. Cancer Sci 2016;107:1013-7.

7. Rosenblatt J, McDermott DF. Immunotherapy for renal cell carcinoma. Hematol Oncol Clin North Am 2011;25:793-812.

8. Van Gool AR, Verkerk R, Fekkes D, et al. Neurotoxic and neuroprotective metabolites of kynurenine in patients with renal cell carcinoma treated with interferon-alpha: course and relationship with psychiatric status. Psychiatry Clin Neurosci 2008;62:597-602.

9. Cella D, Michaelson MD, Bushmakin AG, et al. Healthrelated quality of life in patients with metastatic renal cell carcinoma treated with sunitinib vs interferon-alpha in a phase III trial: final results and geographical analysis. Br J
Cancer 2010;102:658-64.

10. Miljanovic M, Sindik J, Milunovic V, et al. Factor structure and cut-off scores of the Hospital Anxiety and Depression scale (HADS) in a Croatian sample of adult patients suffering from advanced cancer. Psychiatr Danub 2017;29:451-8.

11. Ficarra V, Novella G, Sarti A, et al. Psycho-social wellbeing and general health status after surgical treatment for localized renal cell carcinoma. Int Urol Nephrol 2002;34:441-6.

12. Cella D, Escudier B, Tannir NM, et al. Quality of Life Outcomes for Cabozantinib Versus Everolimus in Patients With Metastatic Renal Cell Carcinoma: METEOR Phase III Randomized Trial. J Clin Oncol 2018;36:757-64.

13. Ter Veer E, van Kleef JJ, Sprangers MAG, et al. Reporting of health-related quality of life in randomized controlled trials involving palliative systemic therapy for esophagogastric cancer: a systematic review. Gastric Cancer 2018;21:183-95.

14. Zaidi S, Hussain S, Verma S, et al. Efficacy of Complementary Therapies in the Quality of Life of Breast Cancer Survivors. Front Oncol 2018;7:326.

15. Zigmond AS, Snaith RP. The hospital anxiety and depression scale. Acta Psychiatr Scand 1983;67:361-70.

16. Tung HY, Chao TB, Lin YH, et al. Depression, Fatigue, and QoL in Colorectal Cancer Patients During and After Treatment. West J Nurs Res 2016;38:893-908.

17. Tripp DA, Verreault P, Tong S, et al. Biopsychosocial impact of prostate cancer and androgen-deprivation therapy. Can Urol Assoc J 2017;11:338-43.

18. Oancea C, Suciu C, Timar B, et al. The reciprocal relationship between coping mechanisms and lung cancer diagnosis: findings of a prospective study. Cancer Manag Res 2018;10:33-40.

19. Sjamsudin E, Maulina T, Cipta A, et al. Assessment of oral cancer pain, anxiety, and quality of life of oral squamous cell carcinoma patients with invasive treatment procedure. Oral Maxillofac Surg 2018;22:83-90.

20. Canil C, Hotte S, Mayhew LA, et al. Interferon-alfa in the treatment of patients with inoperable locally advanced or metastatic renal cell carcinoma: a systematic review. Can Urol Assoc J 2010;4:201-8.

21. Capuron L, Ravaud A, Dantzer R. Early depressive symptoms in cancer patients receiving interleukin 2 and/or interferon alfa-2b therapy. J Clin Oncol 2000;18:2143-51.

22. Choi JS, Kim W, Sohn BK, et al. Association of Changes in Mood Status and Psychosocial Well-Being with Depression During Interferon-Based Treatment for 
Hepatitis C. Psychiatry Investig 2017;14:314-24.

23. Huckans M, Fuller B, Wheaton V, et al. A longitudinal study evaluating the effects of interferon-alpha therapy on cognitive and psychiatric function in adults with chronic hepatitis C. J Psychosom Res 2015;78:184-92.

24. Schiepers OJ, Wichers MC, Maes M. Cytokines and major depression. Prog Neuropsychopharmacol Biol Psychiatry 2005;29:201-17.

25. Capuron L, Dantzer R. Cytokines and depression: the need for a new paradigm. Brain Behav Immun 2003;17 Suppl 1:S119-24.

26. Van Gool AR, Fekkes D, Kruit WH, et al. Serum amino acids, biopterin and neopterin during longterm immunotherapy with interferon-alpha in high-risk melanoma patients. Psychiatry Res 2003;119:125-32.

27. Miller AH, Raison CL. The role of inflammation in depression: from evolutionary imperative to modern treatment target. Nat Rev Immunol 2016;16:22-34.

28. Schenck M, Borgermann C, Jager T, et al. Immunotherapy of metastatic renal cell carcinoma with interleukin-2, interferon-alpha2a and erythropoietin-beta. Urologe A 2007;46:528-34.

29. Belvederi Murri M, Cecere AC, Masotti M, et al. Biopsychosocial predictors of interferon-related depression in patients with Hepatitis C. Asian J Psychiatr 2017;26:24-8.

30. Udina M, Navines R, Egmond E, et al. Glucocorticoid Receptors, Brain-Derived Neurotrophic Factor, Serotonin and Dopamine Neurotransmission are Associated with Interferon-Induced Depression. Int J Neuropsychopharmacol 2016;19:pyv135.

31. Kovacs P, Panczel G, Balatoni T, et al. Social support decreases depressogenic effect of low-dose interferon alpha treatment in melanoma patients. J Psychosom Res 2015;78:579-84.

Cite this article as: Wang Y, Song B, Zhang Y, Li H. Evaluation and predictive factors analyses for patient-selfreported depression, anxiety and quality of life in patients with metastatic renal cell carcinoma underwent interferon- $\alpha$ treatment: a prospective cohort study. Transl Cancer Res 2018;7(6):1460-1473. doi: 10.21037/tcr.2018.11.07
32. Capuron L, Ravaud A, Miller AH, et al. Baseline mood and psychosocial characteristics of patients developing depressive symptoms during interleukin-2 and/or interferon-alpha cancer therapy. Brain Behav Immun 2004;18:205-13.

33. Tadic D, MacLeod C, Cabeleira CM, et al. Age differences in negative and positive expectancy bias in comorbid depression and anxiety. Cogn Emot 2017:1-14.

34. Bien B, Bien-Barkowska K. Objective drivers of subjective well-being in geriatric inpatients: mobility function and level of education are general predictors of self-evaluated health, feeling of loneliness, and severity of depression symptoms. Qual Life Res 2016;25:3047-56.

35. Maatouk I, Herzog W, Bohlen F, et al. Association of hypertension with depression and generalized anxiety symptoms in a large population-based sample of older adults. J Hypertens 2016;34:1711-20.

36. Eigentler TK, Gutzmer R, Hauschild A, et al. Adjuvant treatment with pegylated interferon alpha-2a versus low-dose interferon alpha-2a in patients with high-risk melanoma: a randomized phase III DeCOG trial. Ann Oncol 2016;27:1625-32.

37. Kilbridge KL, Cole BF, Kirkwood JM, et al. Qualityof-life-adjusted survival analysis of high-dose adjuvant interferon alpha-2b for high-risk melanoma patients using intergroup clinical trial data. J Clin Oncol 2002;20:1311-8.

38. McDonnell KK, Bullock LF, Hollen PJ, et al. Emerging issues on the impact of smoking on health-related quality of life in patients with lung cancer and their families. Clin J Oncol Nurs 2014;18:171-81.

39. Weaver KE, Rowland JH, Augustson E, et al. Smoking concordance in lung and colorectal cancer patientcaregiver dyads and quality of life. Cancer Epidemiol Biomarkers Prev 2011;20:239-48. 